\title{
A Wandermonde-like Determinant
}

\author{
BOGDAN C. GRECU
}

\begin{abstract}
We present a determinant which arises from trying to find the extreme points of the unit ball in the space of $n$-homogeneous polynomials defined on the Euclidean plane.
\end{abstract}

The purpose of this note is to present a Wandermonde-like determinant which arises from trying to find the extreme points of the unit ball in the space of $n$-homogeneous polynomials on the Euclidean plane. In order to explain this, let us start with some background information on homogeneous polynomial (see [1] for more details). However, this not needed by the reader merely interested in the determinant announced in the title.

We say that $P$ is an $n$-homogeneous polynomial on a (real or complex) normed space $X$ if there exists an $n$-linear form $B$ on the product $X^{n}$ such that $P(x)=B(x, \ldots, x)$ for every $x$ in $X$. We denote by $\mathcal{P}\left({ }^{n} X\right)$ the space of all continuous $n$-homogeneous polynomials on $X$ endowed with the natural norm $\|P\|=\sup \{|P(x)|:\|x\|=1\}$ and by $\mathcal{L}_{s}\left({ }^{n} X\right)$ the space of continuous symmetric $n$-linear forms on $X$ with the supremum norm. According to the polarization formula [1] for each $P$ there exists a unique $A$ in $\mathcal{L}_{s}\left({ }^{n} X\right)$ such that $P(x)=A(x, x, \ldots, x)$. In general we have

$$
\|P\| \leq\|A\| \leq \frac{n^{n}}{n !}\|P\| .
$$

Furthermore if $X$ is a Hilbert space then $\|P\|=\|A\|$.

A unit vector $x$ in a normed space $X$ is an extreme point of $B_{X}$ if $x$ is not the midpoint of a nontrivial segment lying in $B_{X}$. For instance, in $\mathbb{R}^{2}$ with the Euclidean norm $\left\|\left(x_{1}, x_{2}\right)\right\|=\sqrt{x_{1}^{2}+x_{2}^{2}}$, the unit ball is the disk centred at 0 and of radius 1 and so all the points on the unit circle are extreme.

2000 Mathematics Subject Classification. Primary 11C20, Secondary 46B20.

Key words and phrases. $n$-homogeneous polynomial, determinant. 
It is not true in general that the unit ball of a normed space has extreme points (such an example is $c_{0}$, the space of zero-convergent sequences). However, in dual spaces, and in particular in finite dimensional spaces, extreme points of the unit ball do exist.

\section{The Connection}

Let $H$ denote the Euclidian plane, i.e. the Hilbert space $\mathbb{R}^{2}$ with its Euclidean norm, which does not depend of the choice of the system of orthogonal coordinates. Let $P$ be an $n$-homogeneous polynomial of unit norm and $A$ its associated symmetric $n$-linear form. Let us choose a system of coordinates for $H$ corresponding to the orthogonal basis $\left\{e_{1}, e_{2}\right\}$.

Since $A$ is symmetric we denote by $A\left(x^{k}, x_{k+1}, \ldots, x_{n}\right)$ the fact that $x$ appears $k$ times as an argument for the $n$-linear form $A$. For $0 \leq k \leq n$, let

$$
A_{k}=A\left(e_{1}^{n-k}, e_{2}^{k}\right) .
$$

Then $\left|A_{k}\right| \leq 1$ and the expansion of the polynomial in the basis $\left\{e_{1}, e_{2}\right\}$ is

$$
P\left(x_{1}, x_{2}\right)=\sum_{k=0}^{n} A_{k}\left(\begin{array}{l}
n \\
k
\end{array}\right) x_{1}^{n-k} x_{2}^{k} .
$$

Thus the space of $n$-homogeneous polynomials on $H$ is finite dimensional and so its unit ball has extreme points which we will call extreme polynomials.

When $n=2$, we have

$$
P\left(x_{1}, x_{2}\right)=A_{0} x_{1}^{2}+2 A_{1} x_{1} x_{2}+A_{2} x_{2}^{2} .
$$

By a result of Sundaresan [4], the polynomial of unit norm $P$ is extreme if and only if the two eigenvalues of the matrix $\left(\begin{array}{cc}A_{0} & A_{1} \\ A_{1} & A_{2}\end{array}\right)$ have modulus one. That is to say that $P$ attains its norm at at least two distinct points, i.e. $|P(x)|=\|P\|=1$ for at least 2 points $x$ of unit norm.

The result changes for higher degrees. For instance, in the case $n=3$ there exists a characterization, but there are extreme polynomials that attain their norm at just one point (one example is $\left.P(x)=x_{1}^{3}+3 / 2 x_{1} x_{2}^{2}\right)$, whereas norm attainment at two distinct points still assure the fact that the polynomial is extreme [2].

For $n \geq 4$ characterizations are more difficult to obtain [3]. The case $n=4$ is still solvable, but for $n>4$ only sufficient conditions are 
given, all of them in terms of how many points of norm attainment the polynomial has. When $n$ is odd, norm attainment at $n+1$ points makes the polynomial extreme, whereas for even $n$ the same is true for $n+2$ points of norm attainment. This happens because, provided a suitable coordinate system is chosen, the fact that $|P(x)|=1$ at so many points will exactly determine the two first coefficients of the polynomial, $A_{0}$ and $A_{1}$ and will force the other $n-1$ to satisfy a system of $n-1$ equations, when $n$ is odd and $n$ equations when $n$ is even. The polynomials $P$ will be uniquely determined (and thus extreme) because this system of equations will have unique solution. In both cases, this is done by showing that for every positive integer $l$, and $y_{i} \neq y_{j}$ for $i \neq j$, the following matrix has a nonzero determinant:

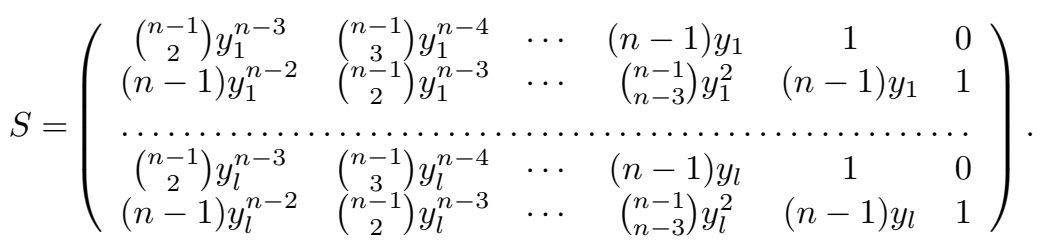

\section{The Determinant}

We will now evaluate the determinant of the above matrix.

Let us define a function $r(y)$ as the determinant of a matrix whose rows but the first two equal those of $S$ and where instead of $y_{1}$, on the first two rows we have a variable $y$. Obviously $r$ is a polynomial of degree $2(n-3)=4(l-1)$ in $y$. It is easily seen that $y_{2}, y_{3}, \ldots, y_{l}$ are zeros for $r$. We have

$$
\begin{aligned}
& r^{\prime}(y)=
\end{aligned}
$$

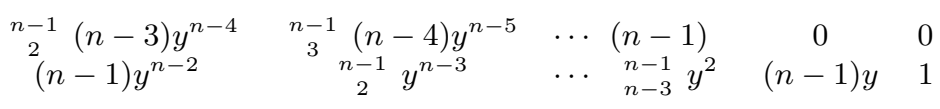

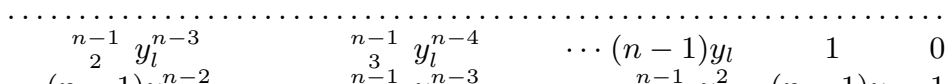

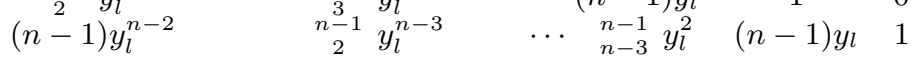

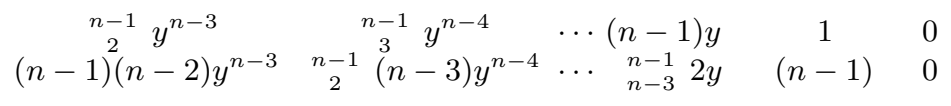

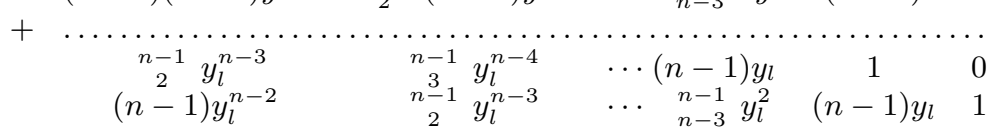


It is obvious that $y_{2}, y_{3}, \ldots, y_{l}$ are also zeros for $r^{\prime}$. Let us go one more step and evaluate $r^{\prime \prime}(y)$. It is a sum of three determinants

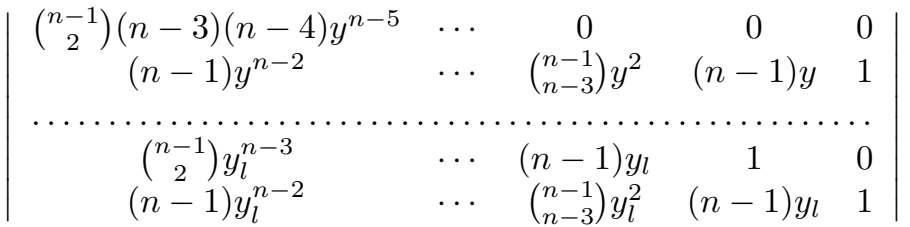

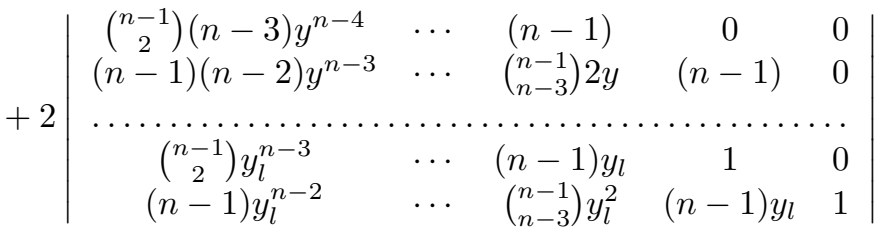

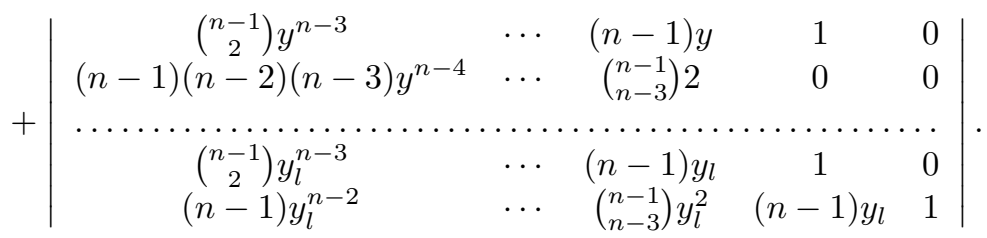

If we replace $y$ by any of the $y_{j}, j \in\{1,2, \ldots, l\}$ we get the value zero for the first and third determinant. Let us show that the same happens to the second one.

In the initial matrix, on the column $k-1$ with $k \in\{2,3, \ldots n-1\}$ we had $\left(\begin{array}{c}n-1 \\ k\end{array}\right) y^{n-k-1}$ on the first row and $\left(\begin{array}{c}n-1 \\ k-1\end{array}\right) y^{n-k}$ on the second row so that in the second determinant above on the column $k-1$ we have $\left(\begin{array}{c}n-1 \\ k\end{array}\right)(n-k-1) y^{n-k-2}=(n-1)\left(\begin{array}{c}n-2 \\ k\end{array}\right) y^{n-k-2}$ on the first row and $\left(\begin{array}{c}n-1 \\ k-1\end{array}\right)(n-k) y^{n-k-1}=(n-1)\left(\begin{array}{c}n-2 \\ k+1\end{array}\right) y^{n-k-1}$ on the second row. On the column $n-2$ we have 0 on the first row and $n-1$ on the second. The last column contains only 0's. Let us divide both of the first two rows by $n-1$ and multiply the first row by $y$, then add the second row to the first one. On the column $k-1$ of the first row we will have

$$
\left(\begin{array}{c}
n-2 \\
k
\end{array}\right) y^{n-k-1}+\left(\begin{array}{l}
n-2 \\
k-1
\end{array}\right) y^{n-k-1}=\left(\begin{array}{c}
n-1 \\
k
\end{array}\right) y^{n-k-1}
$$

for the first $n-3$ rows while on the second last we obtain $0+1=1$ and on the last 0 . Therefore the first row becomes identical to its 
initial form in the expression of $r$ and consequently the determinant will vanish for $y_{2}, y_{3}, \ldots, y_{l}$ so they are all zeros for $r^{\prime \prime}$.

We prove now that $y_{2}, y_{3}, \ldots, y_{l}$ are zeros for $r^{\prime \prime \prime}$. Clearly $r^{\prime \prime \prime}(y)$ is a sum of four determinants. The second row of the first determinant preserves its initial form and so does the first row of the fourth determinant. Therefore the first and the fourth determinants vanish when $y$ is replaced by any of $y_{2}, y_{3}, \ldots, y_{l}$. The sum of the other two determinants is

$$
\begin{aligned}
& D_{1}+D_{2}=
\end{aligned}
$$

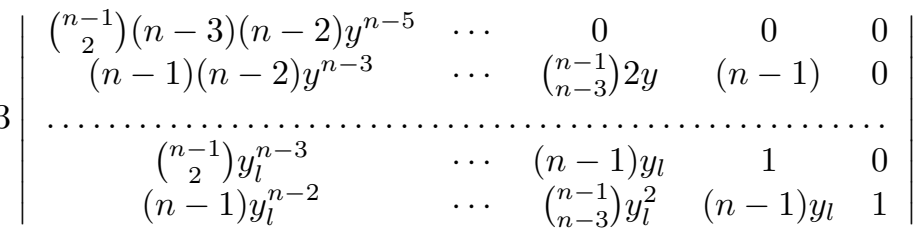

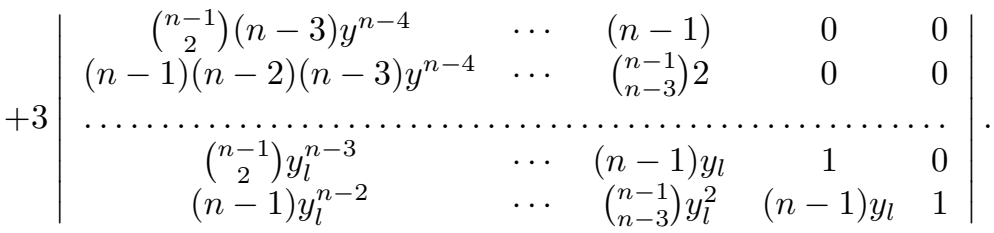

For $D_{1}$, on the column $k-1$, with $k \in\{2,3, \ldots, n-3\}$, on the first row we have

$$
\left(\begin{array}{c}
n-1 \\
k
\end{array}\right)(n-k-1)(n-k-2) y^{n-k-3}=(n-1)(n-2)\left(\begin{array}{c}
n-3 \\
k
\end{array}\right) y^{n-k-3}
$$

and on the second

$$
\left(\begin{array}{l}
n-1 \\
k-1
\end{array}\right)(n-k) y^{n-k-1}=(n-1)\left(\begin{array}{l}
n-2 \\
k-1
\end{array}\right) y^{n-k-1} .
$$

Let us try to bring $D_{2}$ to a nicer form. On the column $k-1$, with $k \in\{2,3, \ldots, n-2\}$ we have $(n-1)\left(\begin{array}{c}n-2 \\ k\end{array}\right) y^{n-k-2}$ on the first row and on the second

$$
\left(\begin{array}{l}
n-1 \\
k-1
\end{array}\right)(n-k)(n-k-1) y^{n-k-2}=(n-1)(n-2)\left(\begin{array}{l}
n-3 \\
k-1
\end{array}\right) y^{n-k-2} .
$$


If we divide the first row by $n-1$ and the second one by $(n-1)(n-2)$ and subtract the second row from the first one, we get

$$
\left(\begin{array}{c}
n-2 \\
k
\end{array}\right) y^{n-k-2}-\left(\begin{array}{c}
n-3 \\
k-1
\end{array}\right) y^{n-k-2}=\left(\begin{array}{c}
n-3 \\
k
\end{array}\right) y^{n-k-2}
$$

on the first $n-4$ columns and 0's on the last three. Extracting $y$ as a factor from the first row and introducing it in the second one we obtain

$$
\begin{aligned}
& 3(n-1)^{2}(n-2) \times
\end{aligned}
$$

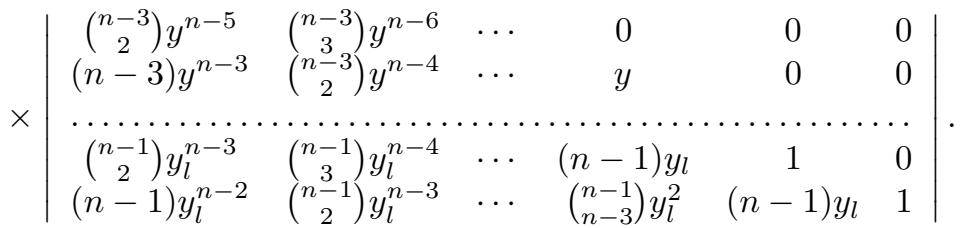

Thus

$$
\begin{aligned}
& \frac{1}{3(n-1)^{2}(n-2)}\left(D_{1}+D_{2}\right)= \\
& \mid \begin{array}{cccccc}
\left(\begin{array}{c}
n-3 \\
2
\end{array}\right) y^{n-5} & \left(\begin{array}{c}
n-3 \\
3
\end{array}\right) y^{n-6} & \ldots & 0 & 0 & 0 \\
(n-2) y^{n-3} & \left(\begin{array}{c}
n-2 \\
2
\end{array}\right) y^{n-4} & \ldots & \left(\begin{array}{c}
n-2 \\
n-3
\end{array}\right) y & 1 & 0 \\
\ldots \ldots \ldots \ldots \ldots \ldots \ldots \ldots \ldots \ldots \ldots \ldots \ldots \ldots \ldots \ldots \ldots \ldots \ldots \ldots \ldots \ldots \ldots \ldots \ldots \ldots \ldots \ldots & \ldots \ldots \ldots
\end{array} \ldots
\end{aligned}
$$

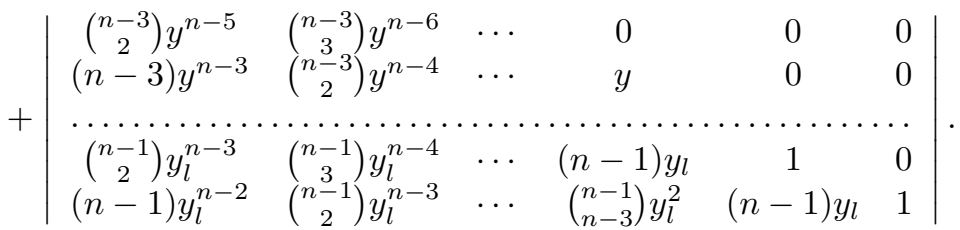

All the rows but the second one are the same so we can add up the second rows of the two determinants. On the column $k-1$, with $k \in\{2,3, \ldots, n-2\}$ we get

$$
\left(\begin{array}{l}
n-2 \\
k-1
\end{array}\right) y^{n-k-2}+\left(\begin{array}{l}
n-3 \\
k-1
\end{array}\right) y^{n-k-2},
$$

on the second last 1 and on the last one we obtain 0 . 
Now let us multiply the first row by $y^{2}$ and add it the second one. On the column $k-1$ with $k \in\{2,3, \ldots, n-3\}$ we obtain

$$
\begin{aligned}
\left(\begin{array}{c}
n-3 \\
k
\end{array}\right) y^{n-k-2}+\left(\begin{array}{l}
n-2 \\
k-1
\end{array}\right) y^{n-k-2}+\left(\begin{array}{l}
n-3 \\
k-1
\end{array}\right) y^{n-k-2} \\
=\left(\begin{array}{c}
n-1 \\
k
\end{array}\right) y^{n-k-2} .
\end{aligned}
$$

On the column $n-3$ we get $(n-2) y+y=(n-1) y$, on the second one 1 and on the last one 0 . Thus the second row we have just obtained is the first row in the initial expression of $r$. All this shows that $D_{1}+D_{2}=0$ when $y$ is replaced by any of $y_{2}, y_{3}, \ldots, y_{l}$.

Therefore $y_{2}, y_{3}, \ldots, y_{l}$ are all zeros of order four for $r$ and thus they are the only zeros that $r$ has. Thus

$$
\operatorname{det} S=\left(\left(\begin{array}{c}
n-1 \\
2
\end{array}\right)^{2}-\left(\begin{array}{c}
n-1 \\
1
\end{array}\right)\left(\begin{array}{c}
n-1 \\
3
\end{array}\right)\right) \prod_{j=2}^{l}\left(y_{1}-y_{j}\right)^{4} \operatorname{det} S_{1}
$$

where $S_{1}$ is the $n-3$-square matrix formed by the last $n-3$ rows and the last $n-3$ columns. The determinant of $S_{1}$ can be evaluated in a similar way. Lowering the dimenssion of the matrix in steps we obtain

$$
\operatorname{det} S=\prod_{k=2}^{n-2}\left(\left(\begin{array}{c}
n-1 \\
k
\end{array}\right)^{2}-\left(\begin{array}{l}
n-1 \\
k-1
\end{array}\right)\left(\begin{array}{l}
n-1 \\
k-2
\end{array}\right)\right) \prod_{1 \leq i<j \leq l}\left(y_{i}-y_{j}\right)^{4} .
$$

Acknowledgements. This article comes from the author's Ph.D. thesis written under the supervision of Dr. Raymond A. Ryan. The author wishes to acknowledge the financial support of a Forbairt Basic Research Grant and of a Postgraduate Fellowship from NUI, Galway. Thanks are also due to Robert Reams for helpful conversations about matrices and determinants.

\section{REFERENCES}

[1] S. Dineen, "Complex Analysis on Infinite Dimensional Spaces", Springer Monographs in Mathematics, Springer-Verlag, London (1999).

[2] B. C. Grecu, Geometry of three-homogeneous polynomials on real Hilbert spaces, J. Math. Anal. Appl. 246 (2000), 217-229.

[3] B. C. Grecu, Geometry of homogeneous polynomials on two-dimernsional real Hilbert spaces, J. Math. Anal. Appl. 293 (2004), 578-588. 
[4] K. Sundaresan, Geometry of Spaces of Quadratic Forms, Inner Product Spaces and Applications. Pitman Research Notes in Mathematics Series 376, Longman, Harlow (1997), 261-265.

Bogdan C. Grecu,

Mathematics Department,

National University of Ireland,

Galway, Ireland

bogdan@wuzwuz.nuigalway.ie

Received on 22 July 2004. 